\title{
RESERVATION-COMPULSORY COMMUTING RAILWAYS: INNOVATION THAT WILL BE MADE POSSIBLE BY UCRT/IPASS
}

\author{
RYO TAKAGI \\ Kogakuin University, Japan
}

\begin{abstract}
Making commuting railways "reservation-compulsory" is a quick method to realise "congestion-free" commute on those railways. This is a simple idea which have generally been rejected as unrealistic. However, the author believes that the advances in technologies recent years have seen is giving the idea a fresh chance to be realised. First, combining the moving block signalling systems and the ideas proposed by the authors to realise ultra-high-frequency train operation, a drastic improvement in capacity on already densely trafficked railway routes will become possible, giving railway operators the ability to provide a wide variety of services that, if properly selected by passengers, will better suit their individual needs. The ultra-high-frequency operation realised in the above way will result in train timetables that are really difficult to use. In addition, a slight imbalance in passenger loading across railway lines, trains or even coaches in a trainset may result in considerable destabilisation of operation. Here comes the need for implementing "compulsory reservation" policy or something close to that, giving the operator the ability to control passenger behaviour to a certain extent. The reservation policy will also give the operator the ability to provide sophisticated and individualised passenger guidance, as proposed by the author in his IPASS proposal. The complicated train timetables will become easy to use through the help of the high-performance individual passenger guidance function of IPASS. Fare collection will also be easy, giving operators the possibility to apply flexible fare structure while maximising passenger benefits. In the paper, the author describes in full detail the concept and possibility of reservation-compulsory commuting railways and reveal that this can be realised by the application of the group of ideas the author has proposed in recent years as part of his "UCRT/IPASS" concept.
\end{abstract}

Keywords: urban railways, ticketing, reservation, passenger guidance, high-frequency scheduling.

\section{INTRODUCTION}

Making commuting railways "reservation-compulsory" is a quick method to realise "congestion-free" commute on those railways. This is a simple idea which have generally been rejected as unrealistic. However, the author believes that the advances in technologies recent years have seen is giving the idea a fresh chance to be realised.

The author has proposed UCRT (Ultra-Convenient Rail Transport), a concept of the railway system that will realise drastically improved convenience from the points of view of individual passengers compared with the conventional railway systems, by means of increase in frequency and diversity of the services on offer. A railway system comprises of subsystems, such as railway infrastructure, railway vehicle, signalling and train control, power supply, communication, ticketing and passenger assistance, etc., each of which is already a very large system itself. This does not change under UCRT; however, the subsystems therein are much more closely integrated than the conventional railway systems, as illustrated in Fig. 1 [1]. This integration is the key to its ability to provide passengers with much more improved railway experience. For example, UCRT passengers can benefit from more direct services without having to change at intermediate stations, while slashing times they have to wait for those services at their originating stations. 


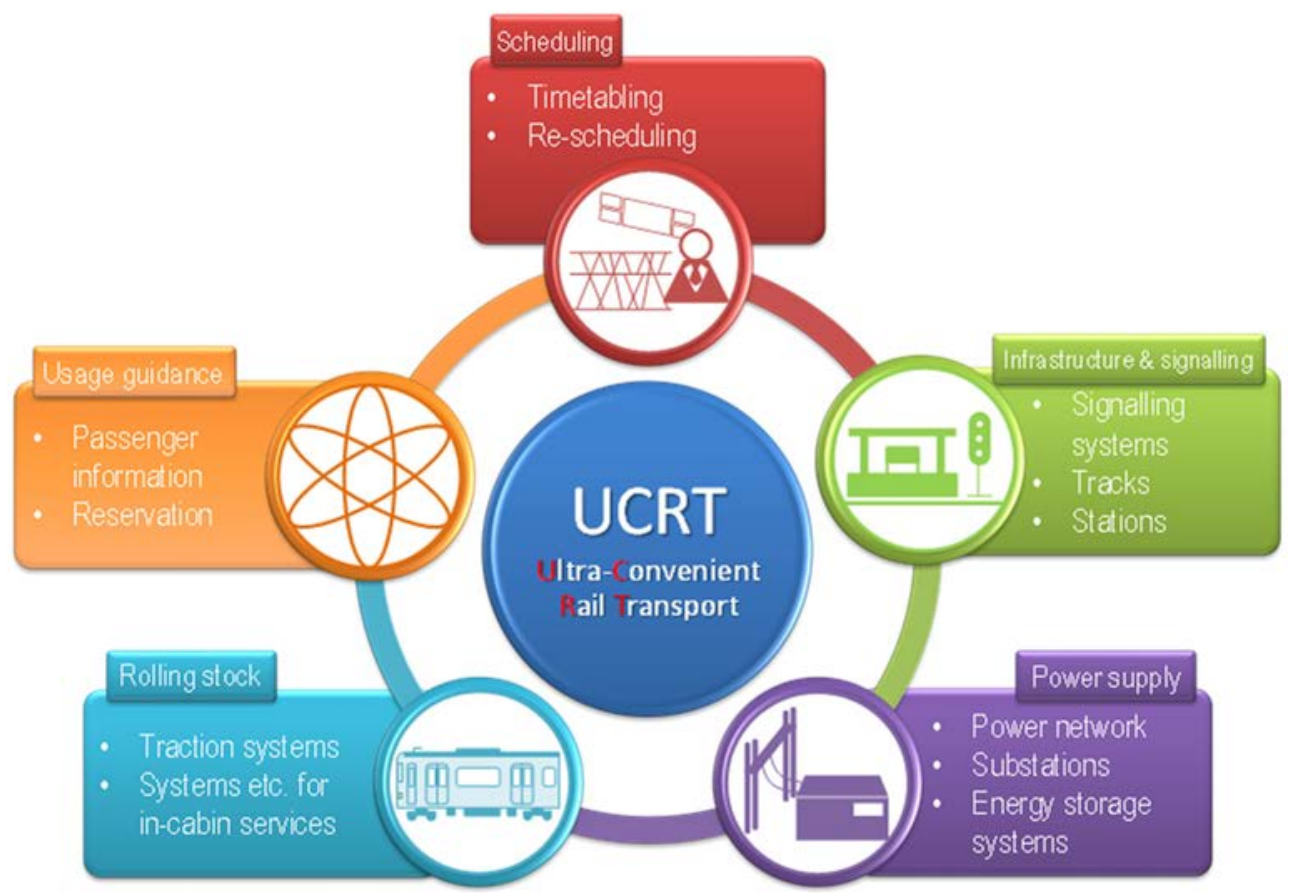

Figure 1: The illustrated concept of UCRT with closer integration of its subsystems [1].

The quick realisation of UCRT on the existing infrastructure will require more complicated timetables, which are much harder to use for passengers, i.e. passengers may find it very difficult to identify the train(s) they have to take to get to their destinations. Therefore, the realisation of far more frequent and far more diverse services, which is one of the major features of UCRT, must come with very advanced passenger assistance. The author has proposed IPASS (Intelligent Passenger Assistance System) back in the 1990s [2] for this purpose. IPASS is an integral and important part of the UCRT concept, which will therefore be referred to as UCRT / IPASS in this paper hereinafter. Using IPASS, passengers can buy tickets and pay the price wherever they want using whatever payment methods of convenience for them and receive guidance information as they want in whatever form they wish.

The author believes that, by applying UCRT/IPASS, the "congestion-free" urban railway system may be realised, in the sense that passengers will not encounter the overcrowded situations on board trains. This will be realised by the combination of ultra-high-frequency train timetabling and reservation-compulsory policy. The reservation policy will enable optimal use of transport capacity through the ability to "control" passengers; it will also give the operator the ability to provide sophisticated and individualised passenger guidance. The complicated train timetables will become easy to use through the help of the highperformance individual passenger guidance function of IPASS. Fare collection will also be easy, giving operators the possibility to apply flexible fare structure while maximising passenger benefits.

In the paper, the author describes in full detail the concept and possibility of reservationcompulsory commuting railways and reveal that this can be realised by the application of the 
group of ideas the author has proposed in recent years as part of his "UCRT/IPASS" concept. First, in Chapter 2, the current situation in Tokyo's urban railways is outlined, which will serve as the basis of the discussions throughout this paper. Together with this, the targets for the reservation-compulsory commuting railways will also be set. Following this, in Chapter 3 , the methods to realise ultra-high-frequency operation will be outlined. In Chapter 4 , the needs for the introduction of "reservation-compulsory" policy will be discussed, together with the discussions over how the added procedure of "reservation" before boarding a train can be simplified. In Chapter 5, the possible challenges in the first phase of the development of reservation-compulsory commuting railways based on the UCRT / IPASS concept will be listed. Finally, in Chapter 6, concluding remarks and future prospects will be shown.

\section{CURRENT SITUATION OF URBAN RAILWAYS IN TOKYO AND TARGETS FOR THE RESERVATION-COMPULSORY COMMUTING RAILWAYS}

Tokyo's commuting railways are infamous for their chronic overcrowding. Although the overall situation has been improved in the recent 20 years or so, the average loading factor of trains during the most crowded one hour on the most crowded section of a major commuting routes in Tokyo frequently reaches 150 to $200 \%$. This naturally means the loading factor of nearly $300 \%$ is still not very uncommon. Typically, 200-metre-long trains are used on these routes, with the frequency of up to 30 trains per hour per direction, and increasing either the train length or the train frequency is generally regarded as prohibitively difficult. Also, during the peak hours, trains tend to become significantly slower for the benefit of shorter headways.

The reservation-compulsory commuting railways are intended to be beneficial to passengers. Therefore, the main target of the development of reservation-compulsory commuting railways is to eliminate this overcrowding on board trains. Then a natural conclusion can be obtained: because of the average loading factor during the peak hours of around $150-200 \%$, the capacity should almost be doubled. This, of course, should be realised without compromising train speed, as current urban railways in Tokyo frequently do.

Also important is that the convenience of passengers is not compromised in any obvious way. For example, there should be an easy way for any passenger to find the right train, and the procedure required to reserve it should be as simple as possible, should take very short period of time and should require very little effort of the passenger.

To summarise, the targets that should be realised by the reservation-compulsory commuting railways are:

[Target 1] Double the transport capacity, without compromising speed; and

[Target 2] Simple and easy-to-use reservation system, together with good guidance.

\section{REALISING DOUBLE CAPACITY}

The author and his research group have a track record of research on increasing capacity. Already, many of the basic ideas are outlined in [3], published nearly 30 years ago.

In this paper, the author classifies the possible ideas and techniques for transport capacity improvements into three categories:

1. Introduction of skip-stop-oriented train timetabling schemes;

2. Maximization of capacity under signalling systems with the 'brick wall' assumptions; and

3. Maximization of capacity using systems without the 'brick-wall' assumptions. 


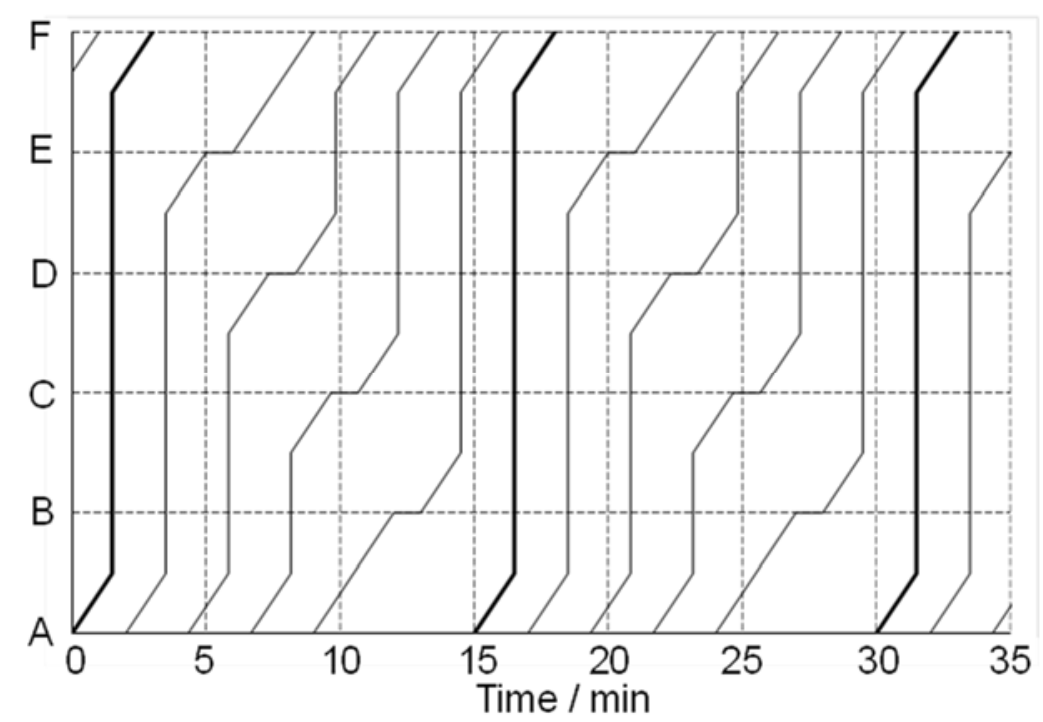

Figure 2: High-frequency timetabling for a high-speed railway using skip-stop timetabling scheme [4]. If the order of trains calling at Stations B through E change, the same capacity cannot be achieved. Although this example is for high speed railways, the same applies to commuting railways as well.

Category 1) ideas can be realised without replacing the conventional fixed block signalling systems. The basic concept behind them is the fact that the headways between trains cruising at a constant speed is much shorter than the headways between trains calling at the same platform in a station [1]. The commonly observed upper limit train frequency of 30 trains per hour per direction, i.e. the shortest average headway between trains of 2 mins, on many major lines in Tokyo comes from the limitation imposed at an intermediate station served by all of these trains while having only one platform per direction. In contrast, when two trains are cruising at the same speed of $60 \mathrm{~km} / \mathrm{h}$ and keeping the headway between them to $1 \mathrm{~min}$, which is half the headway (or double the capacity) of conventional urban railways of Tokyo, the distance between the head of these trains will be $1 \mathrm{~km}$; if the length of the preceding train is $300 \mathrm{~m}$, there still remains a 700-metre space between the tail of the preceding train and the head of the following train. It is possible to design a fixed block signalling system to allow this short separation between running trains.

The problem then is how to let trains stop at stations, since one cannot board a train when it is cruising at a constant speed; to make trains "usable" while making use of short headways during cruising, a sophisticated set of "stopping patterns" of different trains can be used. It is important that, under this timetabling scheme for high-frequency services, the average number of station calls per train will be less than the conventional counterpart. This fact will give the opportunity for capacity increase. Note, however, that the capacity is heavily dependent on the design of the timetable. If the trains are not properly ordered, the capacity increase may not be realised at all, as illustrated in Fig. 2 [4]. This indicates the importance of developing proper techniques for train re-scheduling at the same time.

Category 2) ideas make use of pure moving block (PMB) [5], which can realise the shortest headway under the "brick-wall" assumption. The "brick-wall" assumption is a safety 


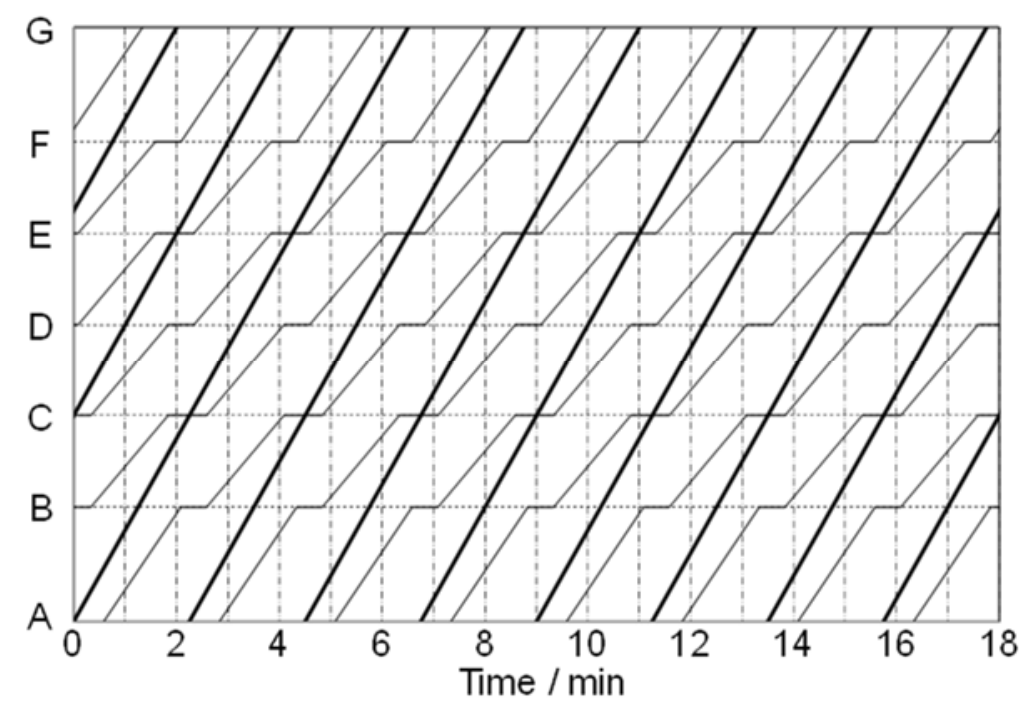

Figure 3: Example train graph of an ultra-high-frequency timetable by applying techniques in Category 2) [4]. This is a timetable with two classes of trains: one "express" train and one "local" train run every 135 seconds. Stations A, C, E and G should have more than one platform. The dwell time of local trains at Stations B, D and $\mathrm{F}$ is uniform at 30 seconds. At Stations $\mathrm{C}$ and $\mathrm{E}$, express trains overtake local trains: the dwell time of the local trains at these stations is 45 seconds and only 15 seconds longer than other intermediate stations. Express trains cruise at the constant speed of $72 \mathrm{~km} / \mathrm{h}$ without having to slow down by coming too close to the local trains.

concept of all practical railway signalling systems. Under this assumption, any train that precedes another must be able to come to a halt with infinite deceleration without compromising safety, i.e. no possibility of collision between this preceding train and a train that follows it on the same railway track arise under such circumstance.

Although PMB is the best system in terms of capacity under this "brick-wall" assumption, its impact on capacity is not thought to be significant, especially compared with the most modern fixed block counterparts. Because the "brick-wall" assumption is clearly a very "conservative" one in terms of safety, efforts to develop systems not based on this assumption, such as relative moving block (RMB) or soft coupling [6], have been made in the hope of realising even shorter headways. In contrast, the author and his research group have sought methods to realise shorter headways under this assumption so the proposals do not become unrealistic. The "synchronisation control" of trains on a railway controlled by PMB [7] is a kind of automatic train operation that will maintain the separation between the adjacent trains to the minimal value allowed under PMB by controlling the acceleration of the train that follows another, thus minimizing headways between these trains. As shown in [6], this technique contributes to the realisation of shorter headways under the practical operating conditions in which unpredictable delays in the departure of trains from platforms occasionally take place. 
It may also be beneficial to point out the issues regarding switches. At the railway switches (or sets of points), trackside equipment such as point machines determine which way a train will proceed. This fact may lead to longer headways at switches. This can be resolved if a technique of realising "on-board switching" can be devised. This on-board switching, however, is not ready at the moment; the author therefore proposed a workaround, so the similar effect can be realised while sticking to the use of the proven trackside switching technique [8].

Combining all these ideas in Category 2), very high frequency timetabling such as shown in Fig. 3 will be possible. However, many stations in urban railways have only one platform per direction, unlike the case of Fig. 3 where every other station has either a passing loop or an additional platform. Under such limitations, application of Category 3) ideas, i.e. signalling systems not based on 'brick-wall' assumption such as soft coupling [6], may be necessary to clear [Target 1] as stated in Chapter 2.

\section{COMPULSORY RESERVATION:}

\section{NEEDS, EFFECTS AND RESOLVING THE INCONVENIENCE}

Even when [Target 1] in Chapter 2 is realised, the capacity has only doubled. As discussed in Chapter 2, more than $200 \%$ of passenger loading is possible in Tokyo's current situation, meaning the capacity is still insufficient to eliminate congestion on board. Fortunately, it is also clear from the discussions in Chapter 2 that this extra-high passenger loading situation will appear only for a fairly short period of time, so once the doubling of capacity is realised, on board congestion can be eliminated if a limited portion of the passengers wanting to use the railway during the most crowded period in a day can be persuaded to shift to other periods. However, various methods tried in the past, such as discounts for off-peak travel, have not been successful for the suppression of such peaks in the demand-time curves.

Also, higher train frequency for the realisation of double capacity will generally mean more unstable operation. A slight imbalance in passenger loading across railway lines, trains or even coaches in a trainset may result in considerable destabilisation of train operation. Passengers should be evenly distributed to different coaches in a trainset, different trains on a railway line and different lines in a network. This can, and should, be addressed to some extent by the proper design of station layouts and timetables, but these are not enough to prevent instability of train operation caused by the unbalanced passenger distributions. The inherent dynamic nature of train movements will also have considerable impacts.

By introducing the compulsory reservation policy, the railway operators can have the methods to control passengers so that these issues are basically eliminated. In the past, reservation was a very costly operation, taking too much time and efforts on both the operators' and passengers' sides, and the reservation-compulsory commuting railway was a totally unrealistic concept. Only when advanced interfacing systems between the railway passengers and the railway system become available will the concept become a possibility. Indeed, as early as 1990s this idea was already part of the proposals of IPASS concept [2].

IPASS aims at the realisation of "ideal" interface between the railway system and the individual passengers. Unlike the conventional interface, using printed-paper-based tickets (sold either at machines or from members of staff either at stations or on trains) and signs (both fixed and variable) and/or vocal announcements either in station premises or on trains, IPASS will let any passenger purchase tickets and pay for it anytime, anywhere using whatever payment method $\mathrm{s} /$ he wants to use, without the need for ticket gates that obstructs smooth movements of passengers in station premises. IPASS also provides very convenient "real-time individual passenger guidance". To facilitate such guidance, it was considered 
necessary that IPASS have the ability of collecting extremely precision information on passengers.

Now, nearly two decades after the proposal of IPASS, powerful handheld communication devices such as smart phones are widely available, which can be used to realise some of the functions proposed in the IPASS concept. However, the ability to collect precision passenger information, such as detection of passengers' current precise positions and their trajectories over the time while they use the railway system, without using ticket gates, is still insufficient. Also, the user interface still needs improvements so that operators can ask all passengers to do reservations before boarding the commuting trains. That said, observing the current state of the development of handheld and/or "wearable" devices, it seems quite natural to expect that, in the future, improvements in the user interface of these devices can solve any issue regarding the inconvenience of reservation.

The procedure of reservation that a passenger undergoes will generally include inputting information on his/her destination. A good guidance of any passenger will not be possible without this information; this fact also encourages compulsory reservation when the timetable is too complicated for general public to find the right service.

It should be noted that the step-by-step introduction method of the compulsory reservation policy should be devised if the policy is to be adopted to the existing railway using conventional interfaces between the passengers and the system. As one research into such technique, the author and his research group discussed the possibility of "reservation without specifying destinations" to ease inconvenience of passengers in the forced reservation procedures; research based on simulations revealed that such "reservation" can be used as well, either if the considerable portion of passengers cooperate with the system in inputting destinations although not required or if the position of passengers can be detected with higher precision.

\section{FIRST MAJOR CHALLENGES IN THE DEVELOPMENT OF} THE RESERVATION-COMPULSORY COMMUTING RAILWAYS

Summarising the discussions in Chapters 3 and 4, the first major challenges to be tackled in the development of the reservation-compulsory commuting railways can be listed.

Realisation of very high frequency timetables such as Fig. 3 will require that the positions of all trains are measured very accurately and transmitted to all subsystems with minimal delays. For example, realising 45 seconds dwell time of a local train while being overtaken by another requires that the trackside point machine starts to operate almost immediately after the tail of the train goes past it. Currently, the errors in train position detection for the existing PMB implementations is around 20 to 100 metres, which is way too long to achieve frequency as high as is shown in Fig. 3.

Generally speaking, detection of train position under PMB is done by measuring the rotating speed of the wheelset and integrating it. However, because of both the possible errors in the wheel diameter used in the calculation and the possible wheel slips, the measured train position will be reset whenever the train passes over one of the position-correcting balises en route. By increasing the number of this kind of balises, the accuracy of train position detection will be improved. However, this straightforward method will come at a relatively high cost, and therefore cheaper and more reliable methods should be sought. For reducing delays in the communication, it would be best to expect developments in other sectors, most probably next generation mobile phone technologies.

For the development of user-friendly interface between the passengers and the railway system, precision data on all passengers' current positions and their trajectories will be highly beneficial. For example, if the railway system can accurately and quickly distinguish whether 
a passenger is on board the train or on the station platform, this information can be used to better utilise capacity. One possible scenario is when, for some reason, a passenger with reservation gets off at the intermediate station without explicitly cancelling the remaining part of his/her reservation: If the train closes the door and start and the passenger is found outside the train, then the seat previously occupied by this passenger is quickly released and assigned to another one, thus improving capacity utilisation.

The biggest obstacle in the realisation of precision measurement of passengers is the concerns for privacy and security. This has already been pointed out in the research into the IPASS concept in the 1990s and no good countermeasure could be devised. To make this technology more widely accepted by the general public, it is necessary to develop more services that people find very useful and which will be made possible only through the collection of such data.

\section{CONCLUSIONS}

In this paper, the UCRT / IPASS concept and the resulting idea of "reservation-compulsory commuting railways" is outlined. Especially, for "reservation-compulsory commuting railways", the current situation of Tokyo's urban railways was used as the basis of the discussion. Even when considering the heavily trafficked situation like that in Tokyo, the author concluded that the possibility exists to develop such a system. As the first of many challenges that should be resolved in the development, the author identified that the precision position detection of trains and passengers is the important step.

The situation in Tokyo, which requires at least doubling of the capacity, is quite in the extreme. However, the author believes it is possible, although the challenges engineers should face are very tough. In contrast, European railways are looking at increasing capacity by adopting a similar set of techniques. For example, "Digital Railway" initiative in UK rail industry [10] states that they aim at "a significant capacity increase" by the realisation of "Digital Railway", a concept that the author regards as very similar to the UCRT / IPASS concept. Interestingly, the same website had in the past a different statement on capacity increase: As of March 2017, they stated clearly " $40 \%$ increase in capacity"; this is a major setback for the project.

This paper describes what is still on the concept level. However, the author believes this paper has pointed out, not only discussion of the possibilities for, but also the likely topics of research and development that may bring in, much needed breakthrough in the increasing the capacity and improving the convenience of railway services and will benefit all future researchers working in this particular field.

\section{ACKNOWLEDGEMENT}

This work has been supported by JSPS KAKENHI Grant Number JP 16K06236.

\section{REFERENCES}

[1] Takagi, R. \& Shimizu, T., Ultra-high frequency train operation for the realization of Ultra-Convenient Rail Transport (UCRT). Proc. 2016 IEEE Int'l. Conf. on Intelligent Rail Transportation (ICIRT), 2016. https://doi.org/10.1109/ICIRT.2016.7588735.

[2] Takagi, R., Haraguchi, T., Naito, M. \& Sone, S., IPASS: Intelligent Passenger Assistance System for Public Transport. Int'l Conf. on "Developments in Mass Transit Systems", London, UK, 1998.

[3] Sone, S., Squeezing capacity out of commuter lines. Dev. Metros 1990, Railw. Gaz. Int., pp. 9-12, 1990. 
[4] Takagi, R., High speed rail - a thought on capacity. Int. Conf. on High Speed Rail: Celebrating Ambition, Birmingham, UK, 2014.

[5] Takeuchi, H., Goodman, C.J. \& Sone, S., Moving block signalling dynamics: performance measures and re-starting queued electric trains. IEE Proc. Electr. Power Appl., 150(4), pp. 483-492, 2003.

[6] Kakuhama, F. \& Takagi, R., Ultra-high frequency train operation using the soft coupling technique: Headway evaluations. Proc. COMPRAIL 2018 (submitted for presentation).

[7] Takagi, R., Synchronisation control of trains on the railway track controlled by the moving block signalling system. IET Electr. Syst. Transp., 2(3), pp. 130-138, 2012.

[8] Takagi, R., Development of very high frequency train operation techniques for the realisation of Ultra-Convenient Rail Transport (UCRT). Rail Lille 2017 - $7^{\text {th }}$ Int'l Conf. on Railway Operations Modelling and Analysis, Lille, France, 2017.

[9] Takagi, R., Reservation-compulsory commuting railways: potentials and challenges. IWTER 2018 (1st IEEJ International Workshop on Transportation and Electric Railway), Bangkok, Thailand, TER18-002, 4 pp., 2018.

[10] http://www.digitalrailway.co.uk/. Accessed on: 9 Apr. 2018. 